\title{
Lesion Detection and Quantitation of Positron Emission Mammography
}

\author{
Jinyi Qi and Ronald H Huesman
}

\begin{abstract}
A Positron Emission Mammography (PEM) scanner dedicated to breast imaging is being developed at our laboratory. We have developed a list mode likelihood reconstruction algorithm for this scanner. Here we theoretically study the lesion detection and quantitation. The lesion detectability is studied theoretically using computer observers. We found that for the zero-order quadratic prior, the region of interest observer can achieve the performance of the prewhitening observer with a properly selected smoothing parameter. We also study the lesion quantitation using the test statistic of the region of interest observer. The theoretical expressions for the bias, variance, and ensemble mean squared error of the quantitation are derived. Computer simulations show that the theoretical predictions are in good agreement with the Monte Carlo results for both lesion detection and quantitation.
\end{abstract}

\section{INTRODUCTION}

Breast imaging using positron emission tomography (PET) has gained increasing interests. Dedicated PET, commonly referred to as positron emission mammography (PEM), has been developed for imaging breast [1], [2], [3], [4]. Comparing to whole body PET scanners, PEM scanners are built with much less number of detector modules and have higher sensitivity.

A rectangular PEM scanner is being developed at our Laboratory. For this scanner, we have developed image reconstruction algorithms, including a filtered backprojection algorithm [4] and a list mode likelihood reconstruction algorithm[5]. In [6] we have also theoretically studied the resolution and noise properties of the list mode reconstruction. Combining these results with computer observer models, we can study the lesion detectability for the list mode likelihood reconstruction and compare the lesion detection with quantitation.

A standard methodology for measuring lesion detectability is the receiver operating characteristic (ROC) study that compares true positive vs. false positive rates for human observers for the task of lesion detection in reconstructed images [7], [8], [9]. However, the human observer ROC study is extremely time-consuming. To overcome this problem, computer observers based on signal detection theory have been developed [10]. Computer observers allow fast evaluation of different algorithms and also provide the possibility of theoretical analysis of lesion detectability. The computer observers that we used are the prewhitening (PW) observer, non-prewhitening (NPW) observer, and region-of-interest (ROI) observer. We particularly focus on the ROI observer because of its unique property of be-

This work was supported by the U.S. Department of Health and Human Services under grant P01 HL25840, by the National Cancer Institute under grant R01 CA 59794, and by the Director, Office of Science, Office of Biological and Environmental Research, Medical Sciences Division of the US Department of Energy under contract DE-AC03-76SF00098.

J. Qi and R.H. Huesman are with the Center for Functional Imaging, Lawrence Berkeley National Laboratory, Berkeley, CA 94720 USA (telephone: 510-4864695, e-mail: \{jqi,rhhuesman\}@lbl.gov). ing capable to achieve the performance of the PW observer with a proper prior as to be shown in Section II and its relationship to lesion quantitation.

This paper is organized as follows. In Section II, we will first review the list mode likelihood reconstruction and its resolution and noise properties; then combine these results with computer observer models. We will also compare lesion detection task with quantitation task and show the difference in the performance of the two tasks. In Section III, the theoretical results are compared with the results from Monte Carlo simulations. The conclusions and discussions are presented in Section IV.

\section{THEORY}

\section{A. List mode likelihood reconstruction}

The log-likelihood function for list mode data is [5]:

$$
L(\boldsymbol{x})=\sum_{k=1}^{K} \log \sum_{j=1}^{N} p\left(i_{k}, j\right) x_{j}-\sum_{j=1}^{N} \varepsilon_{j} x_{j},
$$

where $x_{j}$ is mean activity inside the $j$ th voxel of the unknown image, $p(i, j)$ is the probability of detecting an event from the $j$ th voxel in the $i$ th LOR, $i_{k}$ is the index of the LOR of the $k$ th detection, $\varepsilon_{j} \equiv \sum_{i} p(i, j), K$ is the total number of detections, and $N$ is the total number of image voxels. This model can be modified to include randoms and scatters.

A maximum likelihood (ML) estimate can be found by maximizing (1). A popular ML algorithm for PET reconstruction is the expectation maximization (EM) algorithm [11], [12], [13]. However, the ML solution is very sensitive to the noise in PET data. Hence some form of regularization (or prior function) is needed to reconstruct a reasonable image. The prior function used in [5] is a Gaussian prior whose logarithm is of the form

$$
\beta U(\boldsymbol{x})=\frac{\beta}{2}(\boldsymbol{x}-\boldsymbol{m})^{\prime} \boldsymbol{R}(\boldsymbol{x}-\boldsymbol{m}),
$$

where $\beta$ is the smoothing parameter, $\boldsymbol{m}$ is the estimated mean of the unknown image, and $\boldsymbol{R}$ is a positive definite matrix that defines the neighborhood of the prior.

Combining the likelihood function (1) and the image prior (2), the reconstruction is found as:

$$
\hat{\boldsymbol{x}}=\arg \max _{\boldsymbol{x} \geq 0}[L(\boldsymbol{x})-\beta U(\boldsymbol{x})] .
$$

\section{B. Local impulse response function and covariance}

The likelihood reconstructions are nonlinear and objectdependent because of the Poisson statistics. Thus, the resolution is often studied locally using the local impulse response function. The local impulse response of the $j$ th voxel is defined as 
[14]

$$
\boldsymbol{l}^{j}(\hat{\boldsymbol{x}})=\lim _{\delta \rightarrow 0} \frac{\mathcal{E} \hat{\boldsymbol{x}}\left[\boldsymbol{x}+\delta \boldsymbol{e}_{\boldsymbol{j}}\right]-\mathcal{E} \hat{\boldsymbol{x}}[\boldsymbol{x}]}{\delta}
$$

where $\hat{\boldsymbol{x}}[\boldsymbol{x}]$ denotes the reconstruction of a random data set generated by the source distribution $\boldsymbol{x}, \mathcal{E}$ denotes expectation and $\boldsymbol{e}_{j}$ is the $j$ th unit vector.

We have shown in [6] that (4) can be approximated by

$$
l^{j}(\hat{\boldsymbol{x}}) \approx \boldsymbol{Q}^{\prime} \operatorname{diag}\left[\frac{\lambda_{i}(j)}{\lambda_{i}(j)+\beta \mu_{i}}\right] \boldsymbol{Q} \boldsymbol{e}_{\boldsymbol{j}}
$$

where $\left\{\lambda_{i}(j), i=0, \ldots, N-1\right\}$ is the Fourier transform of the local invariant approximation of the $j$ th column of the Fisher information matrix defined as $\boldsymbol{F} \equiv \boldsymbol{P}^{\prime} \operatorname{diag}\left[\sum_{j=1}^{N} p_{i j} x_{j}\right]^{-1} \boldsymbol{P}$, $\left\{\mu_{i}, \quad i=0, \ldots, N-1\right\}$ is the Fourier transform of $\boldsymbol{R}$ (assuming $R$ is a block circulant matrix), $Q$ and $Q^{\prime}$ represent the Kronecker form of the FFT and its inverse, respectively.

Similarly, the $j$ th column of the covariance matrix can be approximated by

$$
\operatorname{Cov}_{j}(\hat{\boldsymbol{x}}) \approx \boldsymbol{Q}^{\prime} \operatorname{diag}\left[\frac{\lambda_{i}(j)}{\left(\lambda_{i}(j)+\beta \mu_{i}\right)^{2}}\right] \boldsymbol{Q} \boldsymbol{e}_{\boldsymbol{j}} .
$$

\section{Lesion detection using computer observers}

Combining these results with computer observer models, we can derive approximate theoretical expression for SNR of detecting a cancerous lesion in the reconstructed images. Here we use the signal known exactly, background known exactly (SKEBKE) task. One observer is the prewhitening (PW) observer. It corresponds to a likelihood ratio test on a Gaussian distributed image for which the covariance matrix is known and gives the optimal performance for lesion detection with Gaussian noise.

Let $f_{l}$ be the lesion profile and $f_{0}$ the background image. Here we are particularly interested in detection of small lesions, so we can assume the lesion is so small that it hardly changes the noise in the data. Thus, the noise in $\hat{x}$ is independent of the presence of the lesion. Let $\boldsymbol{h}\left(\boldsymbol{f}_{0}+\boldsymbol{f}_{l}\right)$ and $\boldsymbol{h}\left(\boldsymbol{f}_{0}\right)$ denote the mean reconstructions of the image with and without the lesion present, respectively. Then the test statistic of the PW observer is

$$
\eta_{\mathrm{PW}}(\hat{\boldsymbol{x}})=\boldsymbol{z}^{\prime} \boldsymbol{\Sigma}^{-1} \hat{\boldsymbol{x}}
$$

where $\boldsymbol{\Sigma}$ is the covariance matrix of $\hat{\boldsymbol{x}}$ and

$$
\boldsymbol{z} \equiv \boldsymbol{h}\left(\boldsymbol{f}_{0}+\boldsymbol{f}_{l}\right)-\boldsymbol{h}\left(\boldsymbol{f}_{0}\right)
$$

is the observer template.

The observer detection performance can be measured by the signal-to-noise ratio (SNR) of $\eta_{\mathrm{PW}}(\hat{\boldsymbol{x}})$

$$
\begin{aligned}
\mathrm{SNR}_{\mathrm{PW}}^{2} & =\frac{\left[\eta_{\mathrm{PW}}\left(\boldsymbol{h}\left(\boldsymbol{f}_{0}+\boldsymbol{f}_{l}\right)\right)-\eta_{\mathrm{PW}}\left(\boldsymbol{h}\left(\boldsymbol{f}_{0}\right)\right)\right]^{2}}{\operatorname{var}\left[\eta_{\mathrm{NPW}}(\hat{\boldsymbol{x}})\right]} \\
& =\boldsymbol{z}^{\prime} \boldsymbol{\Sigma}^{-1} \boldsymbol{z}
\end{aligned}
$$

By assuming that the covariance around voxel $j$ is locally stationary, $\boldsymbol{\Sigma}$ in (9) can be approximated by

$$
\boldsymbol{\Sigma} \approx \boldsymbol{Q}^{\prime} \operatorname{diag}\left[\frac{\lambda_{i}(j)}{\left(\lambda_{i}(j)+\beta \mu_{i}\right)^{2}}\right] \boldsymbol{Q},
$$

and the $z$ can be approximated by the convolution between the lesion profile and the local impulse response function at the lesion center

$$
\boldsymbol{z} \approx \boldsymbol{Q}^{\prime} \operatorname{diag}\left[\frac{\lambda_{i}(j)}{\lambda_{i}(j)+\beta \mu_{i}}\right] \boldsymbol{Q} \boldsymbol{f}_{l}
$$

Note the above approximations are dependent on the location of the lesion.

Then the $\mathrm{SNR}_{\mathrm{PW}}^{2}$ changes to

$$
\mathrm{SNR}_{\mathrm{PW}}^{2} \approx \frac{1}{\mathrm{~N}} \sum_{\mathrm{i}=0}^{\mathrm{N}-1} \lambda_{\mathrm{i}}(\mathrm{j}) \zeta_{\mathrm{i}}^{2}
$$

where $\left\{\zeta_{i}, i=0, \ldots, N-1\right\}$ is the Fourier transform of the lesion profile $f_{l}$.

When $\eta(\hat{\boldsymbol{x}})$ is normally distributed, the SNR is related to the area under the ROC curve (AUC) by [15]

$$
\mathrm{AUC}=\frac{1}{2}\left[1+\operatorname{erf}\left(\frac{\mathrm{SNR}}{2}\right)\right]
$$

where $\operatorname{erf}(\cdot)$ is the error function.

We have shown in [16] that MAP reconstructions can be approximated by Gaussian distributions, except for the regions where activity is very low. For breast imaging with FDG, we can assume the background is quite uniform. Therefore, the PW observer gives an upper bound on lesion detection in the SKEBKE task. However, the PW observer requires the inverse of the covariance matrix, which is very difficult to compute from the Monte Carlo samples.

A popular observer model that is often used in practice is the non-prewhitening (NPW) observer, which computes the following test statistic [10]

$$
\eta_{\mathrm{NPW}}(\hat{\boldsymbol{x}})=\boldsymbol{z}^{\prime} \hat{\boldsymbol{x}} .
$$

The only difference between (13) and (7) is the deletion of the $\Sigma$, which eliminate the need for the knowledge of the noise. This makes the NPW observer very easy to use in practice.

The SNR of the NPW observer is

$$
\begin{aligned}
\mathrm{SNR}_{\mathrm{NPW}}^{2}= & \frac{\left[\boldsymbol{z}^{\prime} \boldsymbol{z}\right]^{2}}{\boldsymbol{z}^{\prime} \boldsymbol{\Sigma} \boldsymbol{z}} \\
\approx & \left(\sum_{i=1}^{N}\left(\frac{\lambda_{i}(j) \zeta_{i}^{2}}{\lambda_{i}(j)+\beta \mu_{i}}\right)^{2}\right)^{2} \\
& \quad \times\left(N \sum_{i=1}^{N} \frac{\lambda_{i}^{3}(j) \zeta_{i}^{2}}{\left(\lambda_{i}(j)+\beta \mu_{i}\right)^{4}}\right)^{-1} .
\end{aligned}
$$

It can be shown that $\mathrm{SNR}_{\mathrm{NPW}} \leq \mathrm{SNR}_{\mathrm{PW}}$ and the equal sign is achieve if only if the noise is white, i.e., $\boldsymbol{\Sigma}$ is equal to the identity matrix (up to a scalar).

Is there an easy-to-use computer observer model that can achieve the performance of the PW observer for PEM reconstructions? To answer this question, we studied various computer observer models with different prior parameters. The prior parameters we changed are $\beta, \boldsymbol{m}$, and $\boldsymbol{R}$ in (2). We have found 
that a region-of-interest (ROI) observer can achieve the SNR of the PW observer under certain smoothing conditions.

The test statistic of the ROI observer is [17]

$$
\eta_{\mathrm{ROI}}(\hat{\boldsymbol{x}})=\boldsymbol{f}_{l}^{\prime} \hat{\boldsymbol{x}}
$$

It uses the true lesion profile to match with the reconstruction. This differs from (13) in that the NPW observer uses the reconstructed lesion profile. The performance of the ROI observer is

$$
\mathrm{SNR}_{\mathrm{ROI}}^{2}=\frac{\left(\boldsymbol{f}_{\mathrm{l}}^{\prime} \boldsymbol{z}\right)^{2}}{\boldsymbol{f}_{\mathrm{l}}^{\prime} \boldsymbol{\Sigma} \boldsymbol{f}_{\mathrm{l}}}
$$

When we choose the zero-order quadratic prior, i.e., $\boldsymbol{R}=\boldsymbol{I}$ in (2), we can substitute (10) and (11) into the above expression and get

$$
\begin{aligned}
\mathrm{SNR}_{\mathrm{ROI}}^{2} \approx & \left(\sum_{i=0}^{N-1}\left(\frac{\lambda_{i}(j) \zeta_{i}^{2}}{\lambda_{i}(j)+\beta}\right)\right)^{2} \\
& \times\left(N \sum_{i=0}^{N-1} \frac{\lambda_{i}(j) \zeta_{i}^{2}}{\left(\lambda_{i}(j)+\beta\right)^{2}}\right)^{-1}
\end{aligned}
$$

where $\left\{\zeta_{i}, i=1, \ldots, N\right\}$ is the Fourier transform of the lesion profile $\boldsymbol{f}_{l}$.

Let $\beta$ be sufficiently large such that $\beta \gg \lambda_{i}(j)$, we have

$$
\mathrm{SNR}_{\mathrm{ROI}}^{2} \approx \frac{1}{\mathrm{~N}} \sum_{\mathrm{i}=0}^{\mathrm{N}-1} \lambda_{\mathrm{i}}(\mathrm{j}) \zeta_{\mathrm{i}}^{2} \approx \mathrm{SNR}_{\mathrm{PW}}^{2}
$$

Equation (18) shows that when $\beta$ is sufficiently large, the performance of the ROI observer reaches the upper bound set by the PW observer.

In Fig. 1 we plot the performance of the PW, NPW, and ROI observers as a function of smoothing parameter with $\boldsymbol{R}=\boldsymbol{I}$. Clearly, it shows that the $\mathrm{SNR}_{\mathrm{ROI}}$ monotonically increases as $\beta$ increases and reaches the the value of $\mathrm{SNR}_{\mathrm{PW}}$ when $\beta$ is very large, while the $\mathrm{SNR}_{\mathrm{NPW}}$ is always inferior to the $\mathrm{SNR}_{\mathrm{PW}}$. An interesting point to note is that the maximum point of the $\mathrm{SNR}_{\mathrm{NPW}}$ is dependent on the size of the lesion - the larger the lesion is, the larger the optimum $\beta$ is. This poses some difficulty in choosing the optimum smoothing parameter to maximize $\mathrm{SNR}_{\mathrm{NPW}}$ : one has to know the size of the target lesion in order to determine the optimum smoothing parameter. By comparison, it is very easy to optimize the smoothing parameter for $\mathrm{SNR}_{\mathrm{ROI}}$ : just choose a very large $\beta$.

Note that the monotonic property of the $\mathrm{SNR}_{\mathrm{ROI}}$ is only valid for the zero-order quadratic prior. We plot in Fig. 2 the SNRs of the three observers as a function of the smoothing parameter of a first-order quadratic prior. The prior energy function $U(x)$ of the first-order quadratic prior is

$$
U(x)=\frac{1}{2} \sum_{j=1}^{N} \sum_{k \in \mathcal{N}_{j}, k>j}\left(x_{j}-x_{k}\right)^{2},
$$

where $\mathcal{N}_{j}$ is the set of the six nearest neighbors of voxel $j$ in 3D. Clearly, the $\mathrm{SNR}_{\mathrm{ROI}}$ no longer monotonically increases as $\beta$ increases, and it never reaches the value of the PW observer.

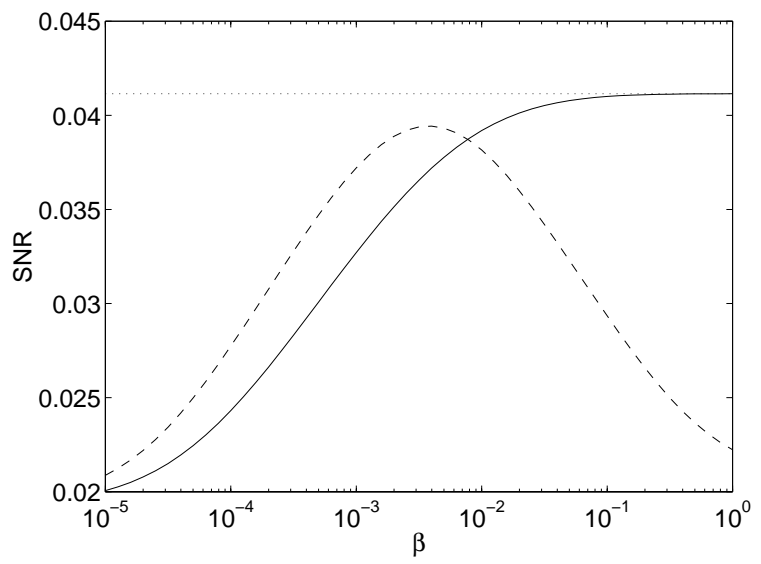

(a)

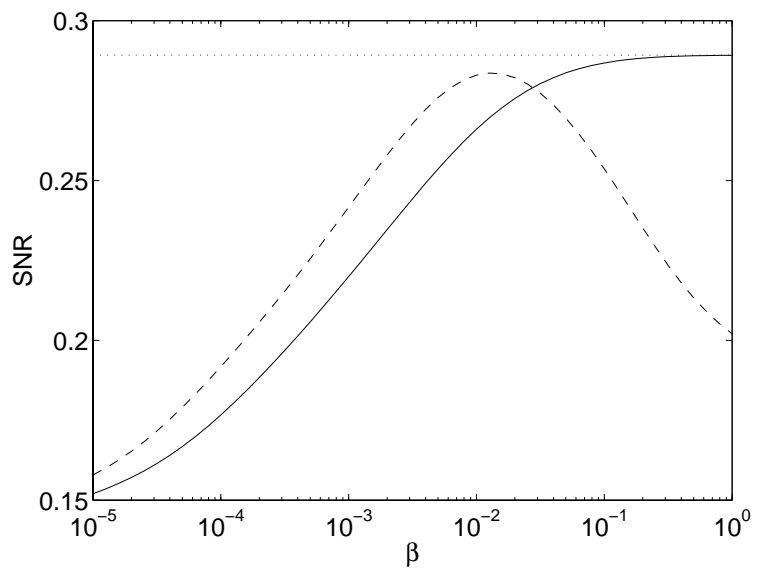

(b)

Fig. 1. Plot of the SNRs of the PW (dotted line), the NPW (dashed line), and the ROI (solid line) observers as a function of the smoothing parameter $\beta$ of the zero-order quadratic prior for a lesion at the center of the FOV: (a) a $2 \mathrm{~mm}$ diameter lesion; (b) a $6 \mathrm{~mm}$ diameter lesion. The contrast of both lesions are one.

\section{Quantitation}

We can also use $\eta_{\text {ROI }}$ to quantify the difference between the lesion uptake and the background activity. In this case, we are more interested in the bias and variance of $\eta_{\mathrm{ROI}}$.

$$
\begin{aligned}
\operatorname{bias}\left(\eta_{\mathrm{ROI}}\right) & \approx \frac{1}{N} \sum_{i=0}^{N-1}\left(\frac{\lambda_{i}(j) \zeta_{i}^{2}}{\lambda_{i}(j)+\beta}\right)-\boldsymbol{f}_{l}^{\prime} \boldsymbol{f}_{l} \\
& =\frac{1}{N} \sum_{i=0}^{N-1}\left(\frac{\beta \zeta_{i}^{2}}{\lambda_{i}(j)+\beta}\right) \\
\operatorname{var}\left(\eta_{\mathrm{ROI}}\right) & \approx \frac{1}{N} \sum_{i=0}^{N-1} \frac{\lambda_{i}(j) \zeta_{i}^{2}}{\left(\lambda_{i}(j)+\beta\right)^{2}} .
\end{aligned}
$$

As a figure of merit for measuring the quality of the quantitation, the ensemble mean squared error (EMSE) can be calculated by

$$
\begin{aligned}
\operatorname{EMSE} & =\left[\operatorname{bias}\left(\eta_{\mathrm{ROI}}\right)\right]^{2}+\operatorname{var}\left(\eta_{\mathrm{ROI}}\right) \\
& =\left[\frac{1}{N} \sum_{i=0}^{N-1}\left(\frac{\beta \zeta_{i}^{2}}{\lambda_{i}(j)+\beta}\right)\right]^{2}
\end{aligned}
$$




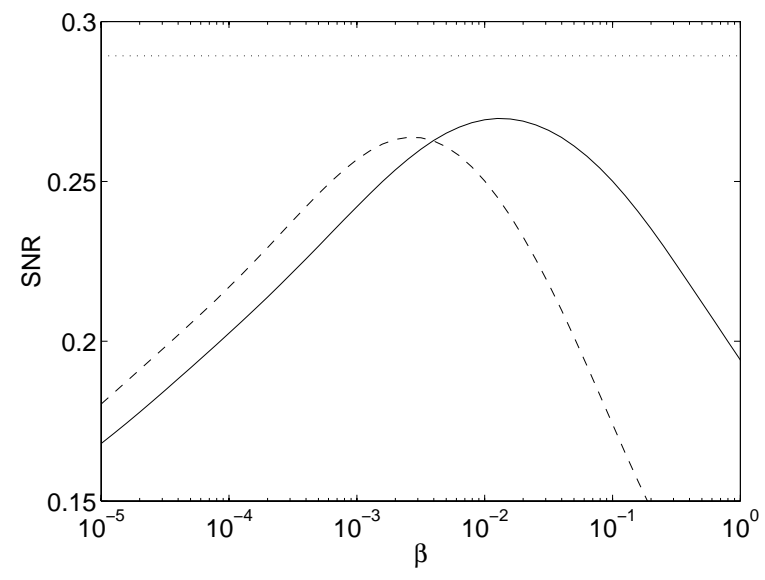

Fig. 2. Plot of the SNRs of the PW (dotted line), the NPW (dashed line), and the ROI (solid line) observers as a function of the smoothing parameter $\beta$ of the first-order quadratic prior for a $6 \mathrm{~mm}$ lesion at the center of the FOV. The contrast of the lesion is one.

$$
+\frac{1}{N} \sum_{i=0}^{N-1} \frac{\lambda_{i}(j) \zeta_{i}^{2}}{\left(\lambda_{i}(j)+\beta\right)^{2}} .
$$

To achieve the minimum EMSE estimate of the lesion uptake, we can evaluate (21) and choose the $\beta$ that gives the minimum value. Fig. 3 shows the plots of the relative EMSE as a function of the smoothing parameter $\beta$ for different lesions. It shows that the relative EMSE reduces when the contrast or the size of the lesion is increased. It is worthy to note the difference between the SNR of lesion detection shown in (17) and the EMSE in (21): while the SNR in (17) is linearly proportional to the contrast of the lesion and always achieves its maximum when $\beta$ is sufficiently large, the shape of EMSE curve and its optimum point are dependent on the lesion contrast - the higher the contrast is, the smaller the optimum $\beta$ is. This indicates the optimum smoothing parameters for detection and for quantitation can be very different.

\section{Monte CARlo Simulations}

We reconstructed 100 computer generated Monte Carlo data sets with 5 different smoothing parameters. Among the 100 data sets, 50 data sets contain purely a uniform background and 50 data sets contain the background with a $6 \mathrm{~mm}$ spherical lesion at the center of the FOV. We ran the ROI observer and the NPW observer over the 100 reconstructions and computed the SNR for both observers. The results are shown in Table I. The standard deviations were computed using the bootstrap method. The results shows that $\mathrm{SNR}_{\mathrm{ROI}}$ monotonically increases as $\beta$ increases and the ROI observer outperforms the NPW observer when $\beta$ is large.

Fig. 4 shows the comparison between the SNR computed from Monte Carlo reconstructions and theoretical predictions. For both observers, the theoretical results lie within the error bar of the Monte Carlo results, except for $\beta=0.00001$. The Monte Carlo results have a positive bias of $1.5 \%$ because of the limited number of reconstructions. After correcting the bias in the Monte Carlo results, the agreement between the theoretical predictions and the Monte Carlo results are even better. The mismatch for $\beta=0.00001$ may be due to: (1) unconvergence

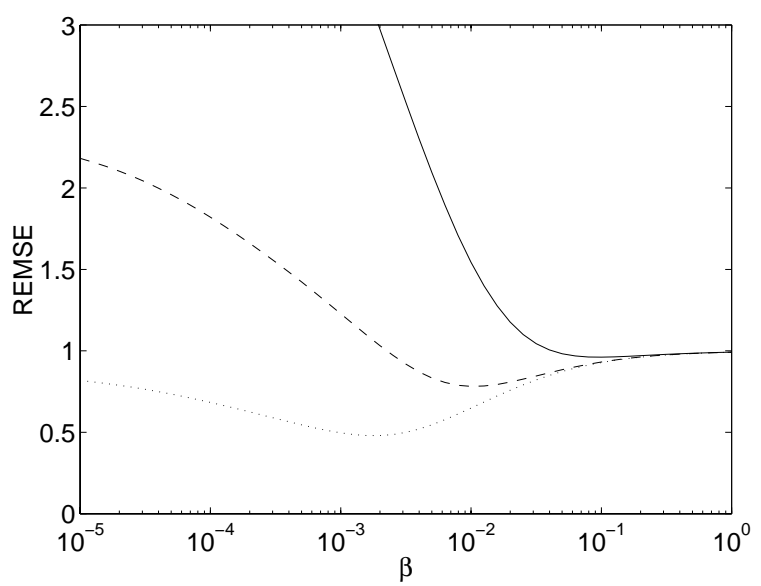

(a)

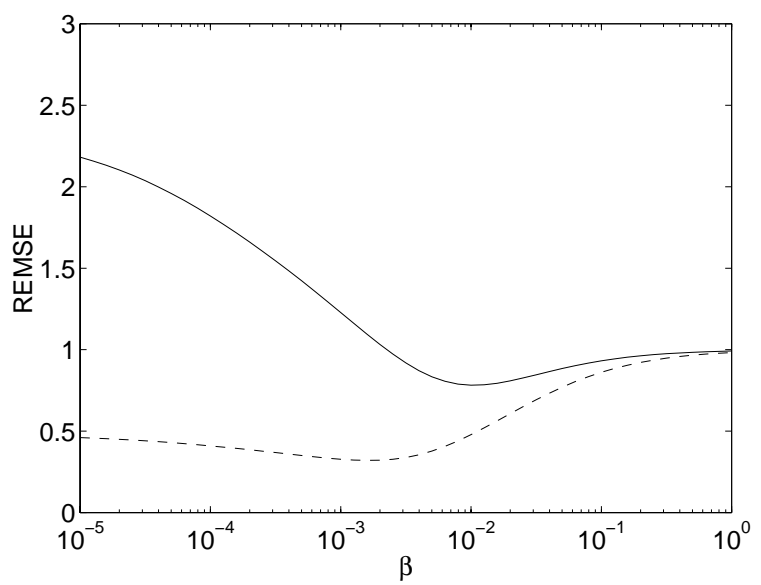

(b)

Fig. 3. Plots of EMSE of the ROI estimator as a function of $\beta$ for a lesion at the center of the FOV. (a) A $6 \mathrm{~mm}$ diameter lesion with contrast equal to 1 (solid line), 3 (dashed line), and 8 (dotted line). (b) A lesion with different diameters: $6 \mathrm{~mm}$ (solid line) and $12 \mathrm{~mm}$ (dashed line). The contrast is 3 .

TABLE I

SNR OF ROI OBSERVER AND NPW OBSERVER FROM MONTE CARLO RECONSTRUCTIONS.

\begin{tabular}{||l|c|c||}
\hline & SNR $_{\mathrm{ROI}}$ & $\mathrm{SNR}_{\mathrm{NPW}}$ \\
\hline$\beta=0.02$ & $1.0187 \pm 0.1198$ & $0.7855 \pm 0.0877$ \\
\hline$\beta=0.005$ & $0.9839 \pm 0.1132$ & $0.8731 \pm 0.0952$ \\
\hline$\beta=0.001$ & $0.9432 \pm 0.1149$ & $0.9508 \pm 0.1026$ \\
\hline$\beta=0.0002$ & $0.8922 \pm 0.1160$ & $0.9404 \pm 0.1145$ \\
\hline$\beta=0.00001$ & $0.8126 \pm 0.0812$ & $0.8623 \pm 0.0935$ \\
\hline
\end{tabular}

of the Monte Carlo reconstructions - when $\beta$ is so small, the convergence of the reconstruction algorithm requires extremely large number of iterations, hence the algorithm are often terminated by the time constraint instead of the convergence criteria; and (2) large noise caused by the insufficient regularization - the theoretical analysis is based on low noise approximations, so the large noise renders the approximations invalidate.

In Fig. 5 we also show the EMSE of the lesion quantitation from the Monte Carlo reconstructions and compare the results to the theoretical predictions. Again, the theoretical predictions match with the Monte Carlo results very well, except for 


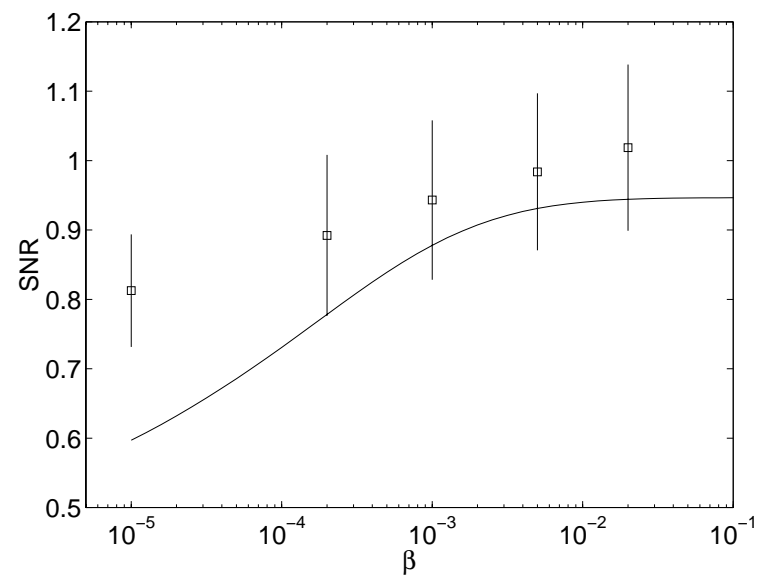

(a)

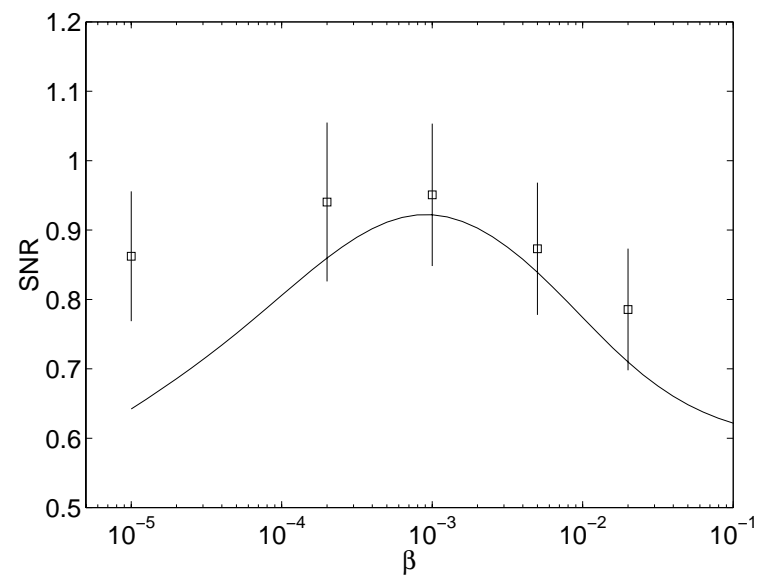

(b)

Fig. 4. Comparison of the SNR from the Monte Carlo reconstructions (squares) and the theoretical predictions (solid lines): (a) the ROI observer and (b) the NPW observer. The error bar were calculated using the bootstrap method.

$\beta=0.00001$ due to the same reasons discussed above. The optimum $\beta$ for achieving the minimum EMSE from the theoretical prediction $\beta=5 \times 10^{-4}$ is also consistent with the Monte Carlo results.

\section{CONCLUSION AND DiscuSSION}

We have theoretically studied the lesion detectability of the list mode likelihood reconstruction for the PEM scanner using computer observers. We have found that the ROI observer can achieve the upper bound of the SKE-BKE detection task by using a zero-order quadratic prior and a sufficiently large smoothing parameter. We have also derived theoretical expressions for the bias, variance, and EMSE of lesion quantitation. Computer simulations show good agreement between the theoretical predictions and the Monte Carlo results for both lesion detection and quantitation. Future work will include extending the study to random lesions and random backgrounds, as well as comparing the computer observer results with human performance.

\section{REFERENCES}

[1] C. Thompson, K. Murthy, Y. Picard, I. Weinberg, and R. Mako, "Positron emission mammography (PEM): A promising technique for detecting breast cancer," IEEE Transactions on Nuclear Science, vol. 42, pp. 10121017, 1995.

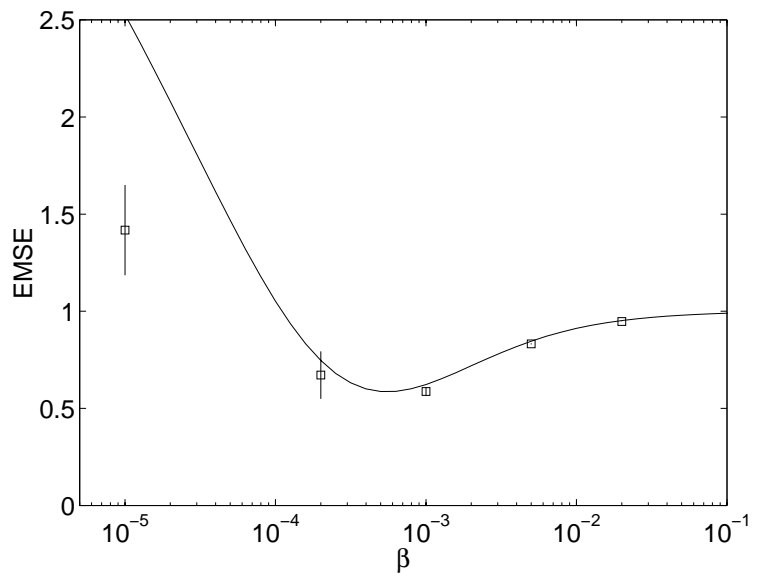

Fig. 5. Comparison of the EMSE from the Monte Carlo reconstructions (squares) and the theoretical predictions (solid lines). The error bar were calculated using the bootstrap method.

[2] R. Freifelder and J. S. Karp, "Dedicated PET scanners for breast cancer," Physics in Medicine and Biology, vol. 42, pp. 2463-2480, 1997.

[3] N. Doshi, Y. Shao, R. Silverman, and S. Cherry, "Design and evaluation of a LSO PET detector for breast cancer imaging," Medical Physics, vol. 27, pp. 1535-1543, 2000.

[4] P. Virador, W. Moses, R. Huesman, and J. Qi, "3D reconstruction in PET cameras with irregular sampling and depth of interaction," IEEE Transactions on Nuclear Science, vol. 48, pp. 1524-1529, 2001.

[5] R. H. Huesman, G. J. Klein, W. W. Moses, J. Qi, B. W. Reutter, and P. R. G. Virador, "List mode maximum likelihood reconstruction applied to positron emission mammography with irregular sampling," IEEE Transactions on Medical Imaging, vol. 19, pp. 532-537, 2000.

[6] J. Qi and R. H. Huesman, "Image properties of list mode likelihood reconstruction for a rectangular positron emission mammograph with DOI measurements," IEEE Transactions on Nuclear Science, vol. 48, pp. 13431349, 2001.

[7] D. R. Gilland, B. M. W. Tsui, C. E. Metz, R. J. Jaszczak, and J. R. Perry, "An evaluation of maximum likelihood-expectation maximization reconstruction for SPECT by ROC analysis," Journal of Nuclear Medicine, vol. 33, pp. 451-457, 1992.

[8] J. Llacer, "Results of a clinical receiver operating characteristic study comparing filtered backprojection and maximum likelihood estimator images in FDG PET studies," Journal of Nuclear Medicine, vol. 34, pp. 11981203, 1993.

[9] T. H. Farquhar, Improved Lesion Detection in Whole Body PET, Ph.D. thesis, University of California, Los Angeles, 1998.

[10] H. H. Barrett, J. Yao, J. Rolland, and K. Myers, "Model observers for assessment of image quality," Proc. Natl. Acad. Sci., vol. 90, pp. 9758$9765,1993$.

[11] A. Dempster, N. Laird, and D. Rubin, "Maximum likelihood from incomplete data via the EM algorithm," Journal of Royal Statistical Society, Series B, vol. 39, pp. 1-38, 1977.

[12] L. Shepp and Y. Vardi, "Maximum likelihood reconstruction for emission tomography," IEEE Transactions on Medical Imaging, vol. 1, pp. 113$122,1982$.

[13] K. Lange and R. Carson, "EM reconstruction algorithms for emission and transmission tomography," Journal of Computer Assisted Tomography, vol. 8, pp. 306-316, 1984.

[14] J. A. Fessler and W. L. Rogers, "Spatial resolution properties of penalizedlikelihood image reconstruction: Spatial-invariant tomographs," IEEE Transactions on Image Processing, vol. 9, pp. 1346-1358, 1996.

[15] H. H. Barrett, T. Gooley, K. Girodias, J. Rolland, T. White, and J. Yao, "Linear discriminants and image quality," Image and Vision Computing, vol. 10, pp. 451-460, 1992.

[16] J. Qi and R. M. Leahy, "Resolution and noise properties of MAP reconstruction for fully 3D PET," IEEE Transactions on Medical Imaging, vol. 19, pp. 493-506, 2000.

[17] H. H. Barrett, "Objective assessment of image quality: Effects of quantum noise and object variability," Journal of the Optical Society of America A, vol. 7, pp. 1266-1278, 1990. 